\title{
Maladies rares: une phase décisive pour les centres de référence
}

\section{Gert Printzen}

Dr med., ancien membre du Comité central de la FMH et responsable du département eHealth - Informatique et documentation médicales /

Produits thérapeutiques, membre de la $\mathrm{Cl}$ Maladies rares

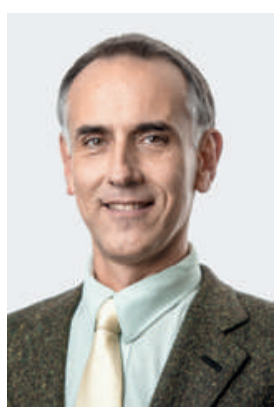

Le concept national maladies rares se trouve actuellement dans une phase décisive de sa mise en œuvre: il s'agit ni plus ni moins de définir les conditions pour les futurs centres de référence nationaux, qui formeront l'un des piliers de la prise en charge des personnes atteintes d'une maladie rare et qui devront notamment assurer la coordination, garantir l'expertise et promouvoir le développement de nouveaux traitements en collaboration avec le milieu de la recherche. Les centres de référence emploieront également des coordinateurs hospitaliers qui seront chargés de soutenir les patients et les professionnels de santé, d'informer et enfin de former le personnel médical. L’Académie suisse des sciences médicales présentera un rapport d’ici l'été.

Les centres de référence doivent intégrer les structures déjà existantes ou prévues et les réseaux existants.

D’après un premier aperçu obtenu à fin février de cette année, l'idée serait de mettre sur pied une coordination nationale dédiée aux maladies rares disposant d'une plateforme virtuelle pour la Suisse alémanique et d'une autre plateforme pour la Suisse romande, toutes deux exploitées par des centres universitaires en collaboration avec les spécialistes et les réseaux de soins spécialisés dans ce domaine.

Pour cela, les centres de référence devront s'appuyer autant que possible sur les structures existantes, aussi bien au niveau universitaire que partout où une expertise spécifique est déjà présente ou en passe de s'établir. Dans le même temps, il s'agira aussi de fixer des normes réalistes, et de tenir compte du fait qu'une fois le diagnostic de maladie rare posé, les structures d'accompagnement médicales et non médicales choisies dépendront essentiellement des symptômes cliniques, par ex. dans le domaine du cœur, des muscles ou du système nerveux ou de plusieurs organes. Dès lors, les institutions et centres de soins de longue durée - en partie non universitaires - spécialisés dans ces groupes de symptômes devront aussi être intégrés dans des réseaux adaptés.
Dans ce contexte, il peut être utile de regarder ce qui se fait à l'étranger. L'exemple français montre qu'une structure imposée d'en haut ne fonctionne même pas de manière satisfaisante dans un Etat centralisé: les Français en sont déjà à la troisième version de leur stratégie nationale. D’une part, les structures déjà existantes, qui doivent être intégrées principalement au plan local et régional et qui sont essentielles pour la prise en charge quotidienne des patients, ont été sous-estimées. Et d'autre part, les personnes concernées souhaitent être traitées par les meilleurs experts du pays. Pour cela, un réseau autorisant une certaine souplesse est primordial. A l'inverse, l'Allemagne est en bonne voie grâce à l'alliance «Nationalen Aktionsbündnis für Menschen mit Seltenen Erkrankungen (NAMSE)»: cette alliance ne dépend pas en soi d'une université; les centres qui en font partie doivent se qualifier pour devenir des centres de référence ou des centres spécialisés, en fonction de leur profil. Le NAMSE bénéficie du soutien de 28 partenaires importants du domaine de la santé, il encourage la recherche et fait le lien entre le corps médical et les instituts de recherche, aussi bien au plan national qu'européen. Le Ministère allemand de l'Education et de la Recherche a d'ores et déjà mis à disposition plus de 110 millions d'Euros (un montant plutôt modeste en regard de l'ensemble des coûts de santé) qui bénéficie directement aux personnes concernées et qui donne les bonnes impulsions. Ce type de collaboration élargie serait une solution souhaitable en Suisse aussi.

\section{Une volonté de coopération partagée par tous} est essentielle au succès d'un projet.

La Communauté d'intérêts Maladies rares (CI Maladies rares) continuera à suivre de manière constructive, critique et collaborative la voie prise par la Suisse, en espérant que ce projet rencontrera un succès équivalent à celui du tunnel de base du Gothard, dans l'intérêt des personnes concernées. Car les personnes atteintes d'une maladie rare ont tout particulièrement besoin d'une prise en charge médicale qui mette avant tout l'accent sur le bien-être des patients à tous les niveaux. 